\title{
An ALMA Dynamical Mass Estimate of the Proposed Planetary-mass Companion FW Tau C
}

\author{
Ya-Lin Wu(i) and Patrick D. Sheehan (10) \\ Steward Observatory, University of Arizona, Tucson, AZ 85721, USA \\ Received 2017 June 26; revised 2017 August 17; accepted 2017 August 20; published 2017 September 8
}

\begin{abstract}
Dynamical mass estimates down to the planet-mass regime can help to understand planet formation. We present Atacama Large Millimeter/submillimeter Array (ALMA) $1.3 \mathrm{~mm}$ observations of FW Tau C, a proposed $\sim 10 M_{\text {Jup }}$ planet-mass companion at $\sim 330$ au from the host binary FW Tau AB. We spatially and spectrally resolve the accretion disk of FW Tau $\mathrm{C}$ in ${ }^{12} \mathrm{CO}(2-1)$. By modeling the Keplerian rotation of gas, we derive a dynamical mass of $\sim 0.1 M_{\odot}$. Therefore, FW Tau C is unlikely a planet, but rather a low-mass star with a highly inclined disk. This also suggests that FW Tau is a triple system consisting of three $\sim 0.1 M_{\odot}$ stars.

Key words: accretion, accretion disks - planets and satellites: individual (FW Tau C) - stars: individual (FW Tau) - techniques: interferometric
\end{abstract}

\section{Introduction}

In the past $\sim 10$ years, many planet-mass companions at wide separations (few to tens of $M_{\text {Jup }}$; tens to hundreds of astronomical units from host stars) have been discovered in direct imaging surveys. Their masses are usually determined by comparing observables like luminosity and effective temperature to predictions from evolutionary and atmospheric models (e.g., Chabrier et al. 2000; Marley et al. 2007; Spiegel \& Burrows 2012; Baraffe et al. 2015), which vary widely depending on the formation pathway (e.g., core accretion versus gravitational instability). As a result, it would be valuable if masses could be dynamically measured. As some of these substellar companions are young $(\lesssim 10 \mathrm{Myr})$ and have features associated with active accretion (e.g., Zhou et al. 2014), their dynamical masses can be measured if their accretion disks can be spatially and spectrally resolved.

Among all of the known wide-separation companions, the proposed planet-mass object FW Tau $\mathrm{C}$ is the prime target to search for a Keplerian-rotating disk. FW Tau C is a tertiary companion located at $\sim 2$ !" 3 projected separation ( $\sim 330$ au) to FW Tau AB, a close binary ( $\sim 0$ !" 08$)$ of nearly equal-mass stars $\left(\sim 0.1 M_{\odot}\right)$ in the $2 \mathrm{Myr}$ Taurus-Auriga star-forming region. FW Tau C was discovered by White \& Ghez (2001) in their survey of binary stars, and its common proper motion was recently confirmed (Kraus et al. 2014). Studies have shown that FW Tau $\mathrm{C}$ has a rather flat near-infrared continuum owing to accretion-induced veiling, as well as many emission lines indicative of outflow and accretion activities (White \& Ghez 2001; Bowler et al. 2014). The accretion disk was previously detected by the Atacama Large Millimeter/submillimeter Array (ALMA) in $1.3 \mathrm{~mm}$ dust continuum (Kraus et al. 2015) and ${ }^{12} \mathrm{CO}(2-1)$ (Caceres et al. 2015), and a 1-2 $M_{\oplus}$ of dust was inferred. Despite these observational efforts, FW Tau C's nature remains enigmatic because strong veiling inhibits accurate mass estimates. Kraus et al. (2014) derived a planetary mass of $10 \pm 4 M_{\text {Jup }}$ from its dereddened $K^{\prime}$ flux, while Bowler et al. (2014) suggested that FW Tau C could be a 0.03-0.15 $M_{\odot}$ brown dwarf or low-mass star embedded in an edge-on disk in order to explain its flat $K$-band spectrum and faint optical and near-infrared brightness.
Accurate mass measurement is therefore key to distinguishing the planet-mass scenario from the stellar-mass scenario. Here, we present the new ALMA $1.3 \mathrm{~mm}$ data from Cycle 3 . With a $\sim 0$.'2 beam and $\sim 0.3 \mathrm{~km} \mathrm{~s}^{-1}$ velocity resolution, we spatially and spectrally resolve the gas disk and derive a dynamical mass of FW Tau C by modeling the Keplerian rotation. Parameters of the FW Tau system are summarized in Table 1.

\section{Methodology}

\subsection{Observations and Data Reduction}

FW Tau was observed with ALMA Band 6 during Cycle 3, on 2016 September 14. During the observations thirty-six $12 \mathrm{~m}$ antennas were available, with baselines ranging from 15 to $3247 \mathrm{~m}$. The Band 6 receiver was configured to have three basebands set up for dust continuum observations, centered at 233.0, 246.0, and $248.0 \mathrm{GHz}$ and each with $2 \mathrm{GHz}$ of bandwidth. The last baseband was configured with $38400.122 \mathrm{MHz}$ channels centered at $230.538 \mathrm{GHz}\left(0.32 \mathrm{~km} \mathrm{~s}^{-1}\right.$ velocity resolution; Hanning smoothed) in order to spatially and spectrally resolve ${ }^{12} \mathrm{CO}(2-1)$ emission from the disk. J0510+1800 was used as the bandpass and flux calibrator, and J0433+2905 was used as the the gain calibrator. The on-source time was $\sim 13$ minutes.

The data were reduced using the ALMA pipeline in the CASA package. We employed one iteration of phase-only selfcalibration to the $2 \mathrm{GHz}$ continuum basebands. The baseband containing $\mathrm{CO}$ emission was much narrower, so it had much lower signal-to-noise ratio in continuum emission and we were unable to obtain good self-calibration solutions. We then CLEANed the calibrated data using the multi-frequency synthesis mode and natural weighting to enhance sensitivity in the images. The continuum map (left panel of Figure 1) has a beam size of 0 ". $29 \times 0$ ". 15 with a position angle (PA) of $160^{\circ} 8$, and an rms of $35 \mu \mathrm{Jy}$ beam $^{-1}$. The CO channel maps (top panel of Figure 3) have a beam size of 0 ". $30 \times 0$ ". 16 with a PA of $160^{\circ} .3$, and a mean rms of $4.5 \mathrm{mJy}^{\text {beam }}{ }^{-1}$ in signalfree channels. The integrated moment-zero and moment-one maps of the $\mathrm{CO}$ emission are shown in Figure 1. 
Table 1

Properties of FW Tau

\begin{tabular}{|c|c|c|c|}
\hline Parameter & FW Tau AB & FW Tau C & References \\
\hline Distance (pc) & \multicolumn{2}{|c|}{$\sim 140$} & 1 \\
\hline Age (Myr) & \multicolumn{2}{|c|}{$\sim 2$} & 2 \\
\hline Separation $(")$ & \multicolumn{2}{|c|}{$\sim 2.3$} & 3,4 \\
\hline $\mathrm{PA}\left({ }^{\circ}\right)$ & \multicolumn{2}{|c|}{$\sim 296$} & 3,4 \\
\hline $\mathrm{SpT}$ & $\mathrm{M} 6 \pm 1$ & $\cdots$ & 3 \\
\hline$A_{V}(\mathrm{mag})$ & $\sim 0.4$ & $\ldots$ & 3 \\
\hline $\log \left(L / L_{\odot}\right)$ & $\sim-1.1^{\mathrm{a}}$ & $\ldots$ & 5 \\
\hline Mass $\left(M_{\odot}\right)$ & $\sim 0.12^{\mathrm{a}}$ & $\sim 0.1$ & 5,6 \\
\hline
\end{tabular}

Note.

${ }^{\mathrm{a}}$ For each component.

References. (1) Kenyon et al. (1994), (2) Kraus \& Hillenbrand (2009), (3) Bowler et al. (2014), (4) Kraus et al. (2014), (5) White \& Ghez (2001), (6) This work

\subsection{Disk Modeling: Continuum}

The continuum emission from FW Tau $\mathrm{C}$ is, at best, only marginally resolved by our observations, so we use a simple geometrical model to fit the data. We assume that the continuum emission traces a uniform brightness disk, with a $1.3 \mathrm{~mm}$ brightness $F_{\nu}$, a radius $R_{\text {disk }}$, and some inclination $i$ and position angle PA. We also allow the centroid of the emission to vary in our fit. We fit the model directly to the continuum visibilities using the Markov Chain Monte Carlo (MCMC) package emcee (Foreman-Mackey et al. 2013).

In order to measure the dust mass of the system, we assume that the continuum emission traces optically thin dust so that we can estimate the disk mass from

$$
M_{\text {disk }}=\frac{F_{\nu} D^{2}}{\kappa_{\nu} B_{\nu}(T)}
$$

(Beckwith et al. 1990). We use standard assumptions of $T=20 \mathrm{~K}$ and $\kappa_{\nu}=2.3 \mathrm{~cm}^{2} \mathrm{~g}^{-1}$ and a distance to FW Tau C of $140 \mathrm{pc}$.

\subsection{Disk Modeling: Keplerian Rotation}

Unlike the $1.3 \mathrm{~mm}$ continuum emission, FW Tau C's gas disk is well resolved by our ${ }^{12} \mathrm{CO}(2-1)$ observations (see Figure 1), including a clear detection of spatially resolved Keplerian rotation. To model the data and determine disk and stellar parameters, we follow the modeling procedure described in Czekala et al. (2015) to fit our channel maps with synthetic channel maps produced from radiative transfer models. Such models can be used to measure disk parameters such as radius and inclination, as well as the stellar (or planetary) mass (Czekala et al. 2015, 2016). Although we follow the procedure outlined by Czekala et al. (2015), we have developed our own codes to run and fit these models to our data set.

We assume that the ${ }^{12} \mathrm{CO}(2-1)$ emission comes from a flared accretion disk, with a density profile described by

$$
\rho(R, z)=\frac{\Sigma(R)}{\sqrt{2 \pi} h(R)} \exp \left[-\frac{1}{2}\left(\frac{z}{h(R)}\right)^{2}\right]
$$

where $R$ and $z$ are defined in cylindrical coordinates, and $\Sigma(R)$ and $h(R)$ are the surface density and disk scale height, respectively. We assume that the disk has a power-law surface density profile:

$$
\Sigma(R)=\Sigma_{0}\left(\frac{R}{R_{0}}\right)^{-\gamma}
$$

and we calculate the $\mathrm{CO}$ column density from this surface density profile as

$$
N_{\mathrm{CO}}(R)=\frac{X_{\mathrm{CO}} \Sigma(R)}{\mu m_{\mathrm{H}}} .
$$

Here, $X_{\mathrm{CO}}=1 \times 10^{-4}$ is the $\mathrm{CO}$ mass abundance fraction, and $\mu=2.37$ is the mean molecular weight. The disk is truncated at an inner radius of 0.1 au and an outer radius of $R_{\text {disk }}$, beyond which the density drops to zero.

We also assume that the disk is in local thermodynamic equilibrium and is vertically isothermal, with a radial temperature profile of

$$
T(R)=T_{0}\left(\frac{R}{1 \mathrm{au}}\right)^{-q} .
$$

Under these assumptions, the scale height in the disk is set by the balance of thermal pressure and gravity such that

$$
h(R)=\left(\frac{k_{b} R^{3} T(R)}{G M_{*} \mu m_{\mathrm{H}}}\right)^{1 / 2}
$$

where $k_{b}$ is the Boltzmann constant, and $G$ is the gravitational constant.

Finally, rotation in the disk is assumed to be Keplerian, with an azimuthal velocity of

$$
v_{k}=\sqrt{\frac{G M_{*}}{r}} .
$$

We assume that the velocities in the radial and vertical directions are zero. We also include microturbulent line broadening, which we assume is uniform throughout the disk, with a value of $\xi$ in units of $\mathrm{km} \mathrm{s}^{-1}$. Finally, we allow the star to have a systemic velocity, $v_{\text {sys }}$, which Doppler shifts the velocity center away from zero.

In all, the density, temperature, and velocity structure of the system are described by the following parameters: $M_{\text {disk }}, R_{\text {disk }}$, $\gamma, T_{0}, q, \xi, M_{*}$, and $v_{\text {sys. }}$. We also allow the viewing geometry of the system, the inclination, and position angle to vary. In our model, the position angle of the disk is defined as the angle east of north of the projection of the disk angular momentum vector on the sky and ranges from $0^{\circ}$ to $360^{\circ}$. As our detection of $\mathrm{CO}$ $(2-1)$ is not high sensitivity, we allow the inclination of the fit to range from $0^{\circ}$ to $90^{\circ}$. With higher sensitivity observations, however, it may be possible to distinguish between $i<90^{\circ}$ and $i>90^{\circ}$ (e.g., Rosenfeld et al. 2013; Czekala et al. 2015). Initially, we center the $\mathrm{CO}$ data based on the results of our geometrical continuum modeling, but allow for a small deviation from that centering in our model.

We fix the distance to the disk at $140 \mathrm{pc}$ (see Table 1). The uncertainty in the distance, however, translates linearly into an uncertainty on the measured stellar mass (e.g., Czekala et al. $2015,2016)$, so we add the uncertainty on the distance estimate in quadrature with the uncertainty derived from our fit. Here, we assume a conservative distance uncertainty of $20 \mathrm{pc}$ following Czekala et al. (2016).

We use the $3 \mathrm{D}$ radiative transfer modeling package RADMC3D (Dullemond 2012) to calculate the level populations in each 

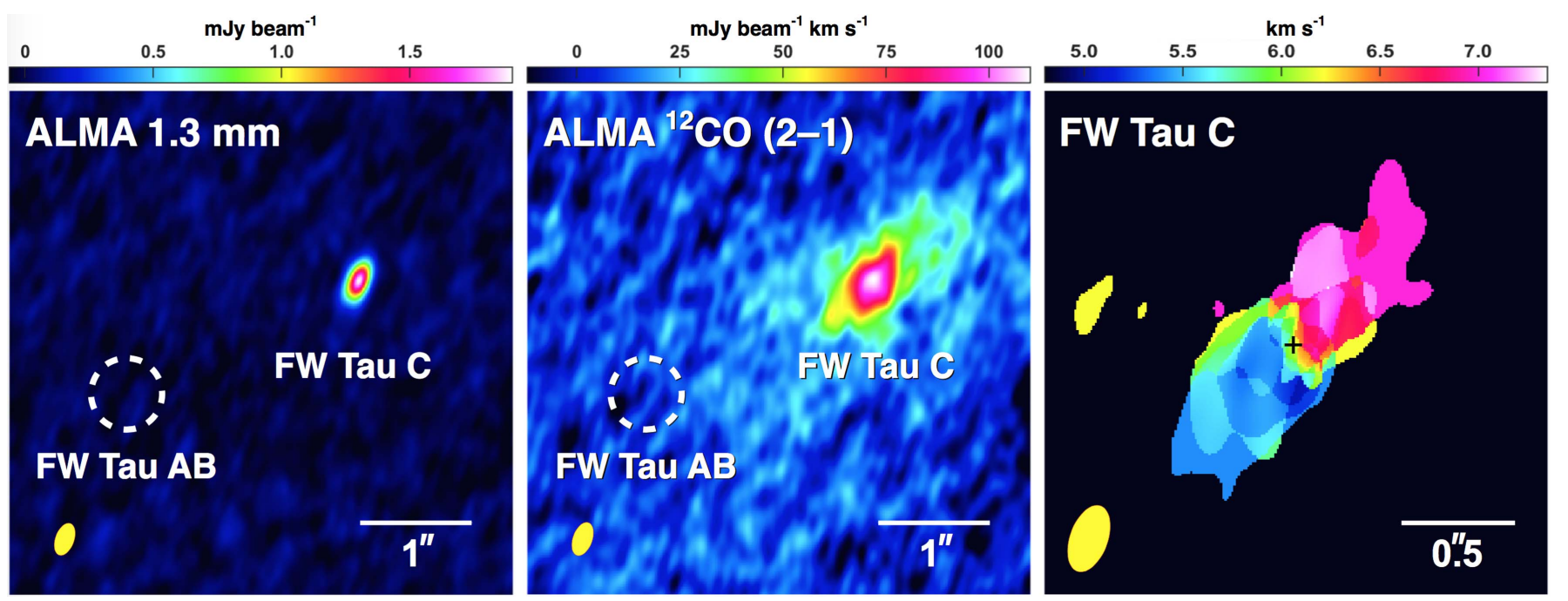

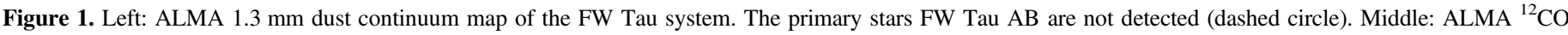

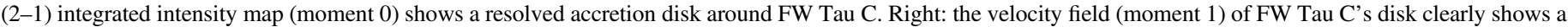
Keplerian rotation. The black cross marks the center of the disk in $1.3 \mathrm{~mm}$ continuum. Beam size $\sim 0$ !. $^{\prime \prime} 9 \times 0$." 16 and PA $\sim 160^{\circ}$. North is up and east is left.

cell and produce synthetic ${ }^{12} \mathrm{CO}(2-1)$ channel maps for a given set of model parameters. Those synthetic channel maps are Fourier transformed and fit directly to the visibilities using the MCMC fitting package emcee (Foreman-Mackey et al. 2013), with uniform priors for all parameters.

Although these models are computationally expensive, they can be run on powerful supercomputers so that the computations are spread out over a large number of central processing units (CPUs). For this particular instance, we run the fit over 128 CPUs on the University of Arizona El Gato supercomputer, and the modeling took a few days to converge. The models were determined to be converged when the emcee walkers had reached a steady state with measured best-fit values changing minimally over a large number of steps.

\section{Results}

Figure 1 shows the $1.3 \mathrm{~mm}$ dust continuum, the ${ }^{12} \mathrm{CO}(2-1)$ integrated intensity map (moment 0 ), and the intensity-weighted velocity map (moment 1 ) of the FW Tau C disk. Similar to Kraus et al. (2015) and Caceres et al. (2015), no signal is found from the close binary $\mathrm{FW}$ Tau $\mathrm{AB}$, and the $3 \sigma$ upper limit for a unresolved source suggests a dust mass $\lesssim 0.07 M_{\oplus}$. It is known that close binaries can shorten disk lifetimes (e.g., Cieza et al. 2009), so the disk around FW Tau AB may be depleted already. For the companion FW Tau C, its dust disk is compact and likely unresolved; in contrast, the gas disk is more extended and clearly shows a Keplerian rotation.

Figure 2 is the position-velocity diagram constructed along the major axis of the gas disk. On top of the PV diagram we also plot the Keplerian rotation curves for $10 M_{\text {Jup }}$ and $0.1 M_{\odot}$ objects. It is clear that the velocity profile of the FW Tau C disk is incompatible with the $10 M_{\text {Jup }}$ rotation curve, but more consistent with that of a $0.1 M_{\odot}$ star. Our disk modeling (see Figure 3 and Table 2) also indicates that FW Tau C's mass is $\sim 0.1 M_{\odot}$, about 10 times higher than the $10 M_{\text {Jup }}$ suggested by Kraus et al. (2014). Hence, FW Tau C is not a planetary-mass object, but is rather a low-mass star with an inclined disk, as suggested by Bowler et al. (2014). The high mass of FW Tau C is also in agreement with some features in its spectrum that closely resemble $\mathrm{T}$ Tauri stars (Bowler et al. 2014). Our

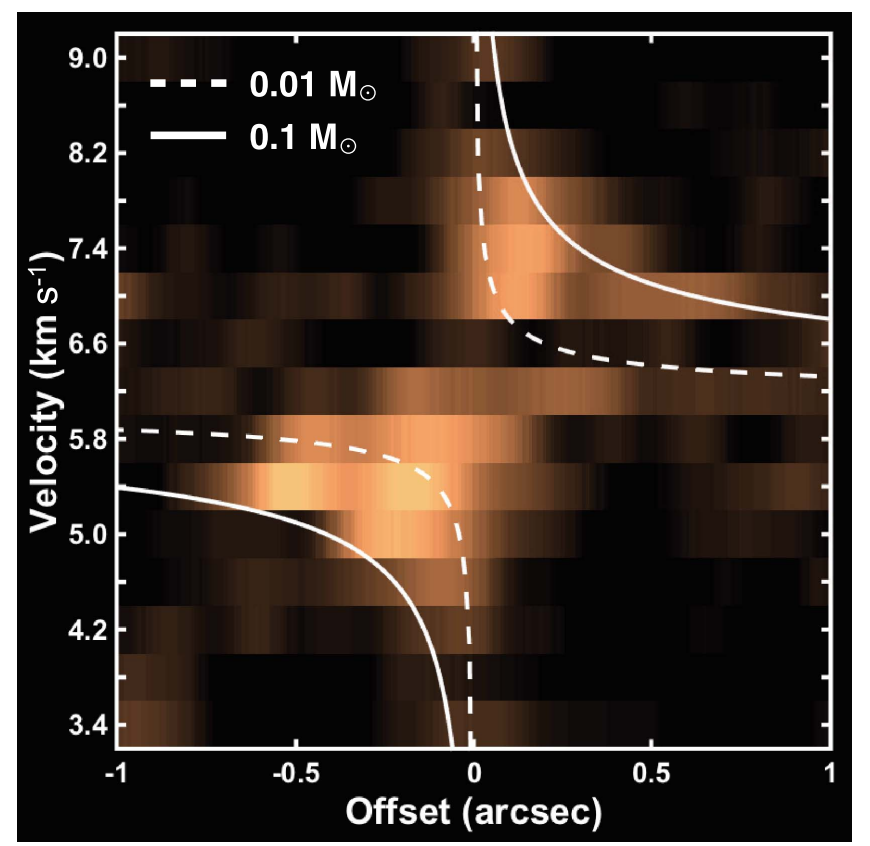

Figure 2. Position-velocity diagram constructed along the major axis of the FW Tau C disk. Keplerian rotation curves for a $0.01 M_{\odot}\left(\sim 10 M_{\text {Jup }}\right)$ planet and a $0.1 M_{\odot}$ star are also plotted. FW Tau C is more consistent with a $0.1 M_{\odot}$ star.

Table 2

FW Tau C Disk Properties

\begin{tabular}{lc}
\hline \hline Parameter & Value \\
\hline$M_{*}\left(M_{\odot}\right)$ & $0.098 \pm 0.015$ \\
$M_{\text {disk,dust }}\left(M_{\oplus}\right)$ & $1.15 \pm 0.01$ \\
$M_{\text {disk,gas }}\left(M_{\oplus}\right)$ & $0.58 \pm 0.13$ \\
$R_{\text {disk }}(\mathrm{au})$ & $141.9 \pm 6.6$ \\
$\gamma$ & $1.63 \pm 0.14$ \\
$T_{0}(\mathrm{~K})$ & $62.1 \pm 34.6$ \\
$q$ & $0.14 \pm 0.10$ \\
$\xi\left(\mathrm{km} \mathrm{s}^{-1}\right)$ & $0.65 \pm 0.08$ \\
$v_{\text {sys }}\left(\mathrm{km} \mathrm{s}^{-1}\right)$ & $6.10 \pm 0.02$ \\
$i\left(^{\circ}\right)$ & $62.8 \pm 2.4$ \\
$\mathrm{PA}^{\circ}\left(^{\circ}\right)$ & $40.6 \pm 1.5$ \\
\hline
\end{tabular}



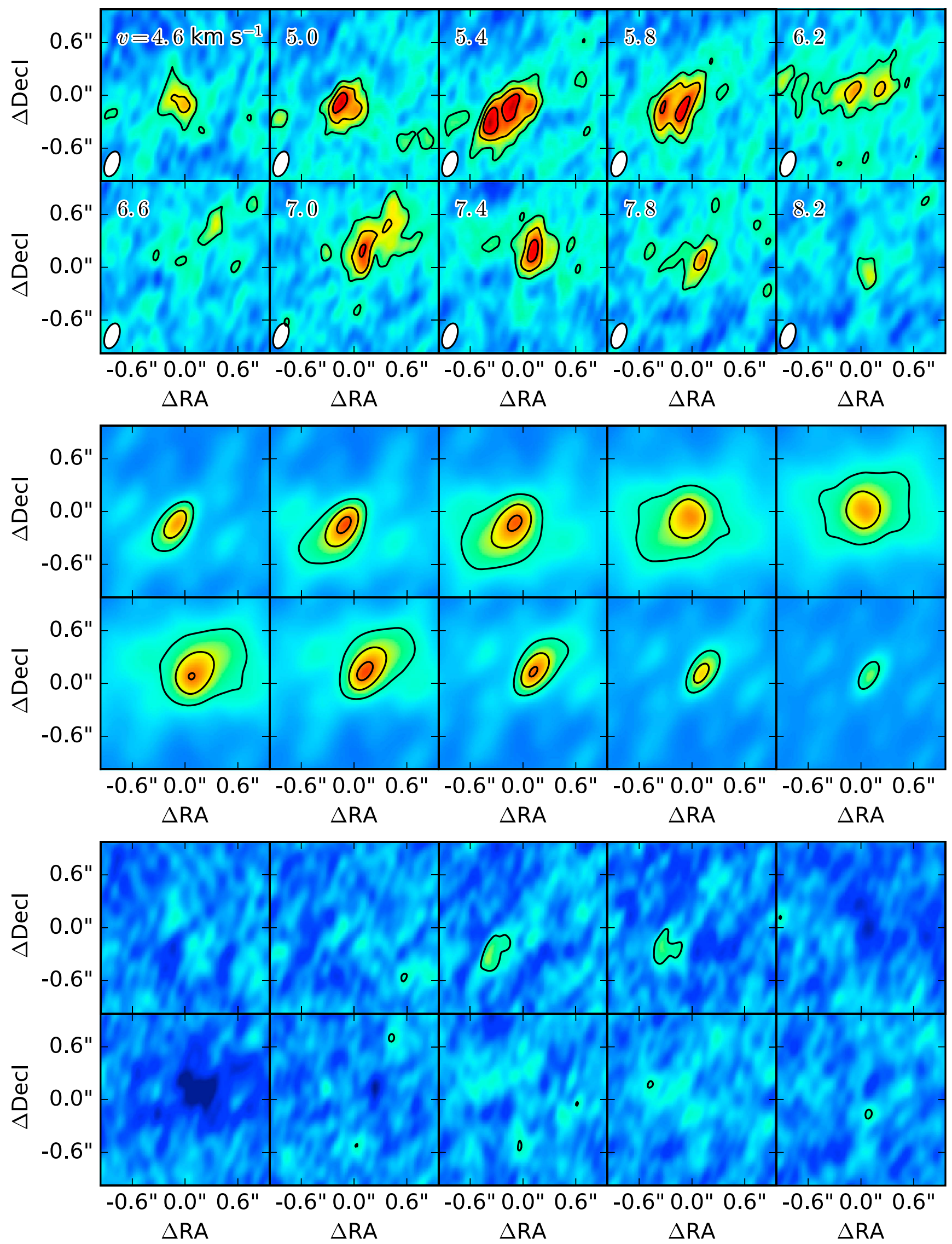

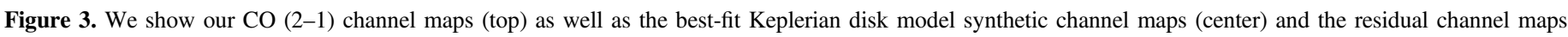

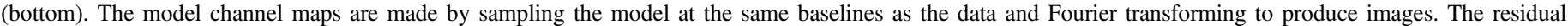

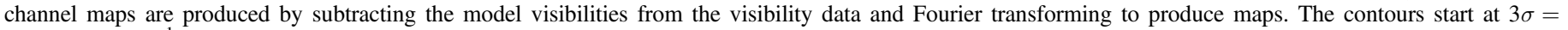
$13.5 \mathrm{mJy} \mathrm{beam}^{-1}$ and are at intervals of $3 \sigma$. 
observations therefore suggest that FW Tau is a young triple system in which each of the stars has a mass of $\sim 0.1 M_{\odot}$.

Searches for accretion disks in millimeter around directly imaged planet-mass companions have not yielded positive results (e.g., Isella et al. 2014; Bowler et al. 2015; MacGregor et al. 2017; Ricci et al. 2017; Wolff et al. 2017; Wu et al. 2017), although the disk around the free-floating planet OTS 44 has recently been imaged (Bayo et al. 2017). Current flux upper limits imply that these wide companion disks might have $<0.1 M_{\oplus}$ of dust, which in turn could imply a very short disk lifetime. Future deep imaging down to a sublunar mass regime is perhaps needed to detect these disks, or place stringent constraints on their masses, and further elucidate the mass growth history of the planetary-mass companions at wide orbits.

Finally, we summarize our disk modeling results as below and also in Table 2. Modeling the FW Tau C's dust disk as a circular Gaussian, we find that it has a $1.3 \mathrm{~mm}$ flux of $\sim 2.06 \mathrm{mJy}$, which equates to a dust mass of $\sim 1.15 M_{\oplus}$ and is consistent with previous measurements in Kraus et al. (2015) and Caceres et al. (2015). For the gas disk, our radiative transfer modeling, as demonstrated in Figure 3, finds that the gas disk has a radius of $\sim 140$ au, inclination of $\sim 63^{\circ}$, PA of $\sim 41^{\circ}$, systemic velocity of $\sim 6.1 \mathrm{~km} \mathrm{~s}^{-1}$, and gas mass of $\sim 0.58 M_{\oplus}$. Although recent studies have shown a low gas-to-dust ratio in protoplanetary disks (e.g., Williams \& Best 2014; Ansdell et al. 2016), we caution that with a single optically thick $\mathrm{CO}$ line, we are very likely to underestimate the true gas mass by a factor of few or even orders of magnitude (e.g., Yu et al. 2017). As Yu et al. (2017) pointed out, to accurately derive the gas mass, one should observe low-transition lines to ameliorate the optical-depth effects and should also use multiple $\mathrm{CO}$ isotopologues to characterize the chemical depletion and abundance variation across the disk.

\section{Summary}

FW Tau C is a wide-orbit companion at $\sim 330$ au from the close binary FW Tau AB that has been suggested to have a planet-like mass of $10 M_{\text {Jup }}$. Here, we have used ALMA to detect FW Tau C's accretion disk in a $1.3 \mathrm{~mm}$ dust continuum and ${ }^{12} \mathrm{CO}(2-1)$ emission. We find that the gas motion is both spatially and spectrally resolved and clearly follows Keplerian rotation, enabling a dynamical mass estimate of the central object. We show that FW Tau C's mass is in fact $\sim 0.1 M_{\odot}$, so it is more likely a low-mass star embedded in an inclined accretion disk.

We thank the referee for very helpful comments. We thank Laird Close, Johanna Teske, Yu-Cian Hong, and Jing-Hua Lin for discussions. Y.-L.W. is supported by the NASA Origins of Solar Systems award and the TRIF fellowship. This Letter makes use of the following ALMA data: ADS/JAO. ALMA\#2015.1.00773.S. ALMA is a partnership of ESO (representing its member states), NSF (USA) and NINS (Japan), together with NRC (Canada), NSC and ASIAA (Taiwan), and KASI (Republic of Korea), in cooperation with the Republic of Chile. The Joint ALMA Observatory is operated by ESO, AUI/NRAO, and NAOJ. The National Radio Astronomy Observatory is a facility of the National Science Foundation operated under cooperative agreement by Associated Universities, Inc.

\section{ORCID iDs}

Ya-Lin Wu (iD https://orcid.org/0000-0002-4392-1446

Patrick D. Sheehan (iD https://orcid.org/0000-0002-9209-8708

\section{References}

Andrews, S. M., Rosenfeld, K. A., Kraus, A. L., \& Wilner, D. J. 2013, ApJ, 771,129

Ansdell, M., Williams, J. P., van der Marel, N., et al. 2016, ApJ, 828, 46

Baraffe, I., Homeier, D., Allard, F., \& Chabrier, G. 2015, A\&A, 577, A42

Bayo, A., Joergens, V., Liu, Y., et al. 2017, ApJL, 841, L11

Beckwith, S. V. W., Sargent, A. I., Chini, R. S., \& Guesten, R. 1990, AJ, 99, 924

Bowler, B. P., Andrews, S. M., Kraus, A. L., et al. 2015, ApJL, 805, L17

Bowler, B. P., Liu, M. C., Kraus, A. L., \& Mann, A. W. 2014, ApJ, 784, 65

Bowler, B. P., Liu, M. C., Kraus, A. L., Mann, A. W., \& Ireland, M. J. 2011, ApJ, 743, 148

Caceres, C., Hardy, A., Schreiber, M. R., et al. 2015, ApJL, 806, L22

Chabrier, G., Baraffe, I., Allard, F., \& Hauschildt, P. 2000, ApJ, 542, 464

Cieza, L. A., Padgett, D. L., Allen, L. E., et al. 2009, ApJL, 696, L84

Czekala, I., Andrews, S. M., Jensen, E. L. N., et al. 2015, ApJ, 806, 154

Czekala, I., Andrews, S. M., Torres, G., et al. 2016, ApJ, 818, 156

Dullemond, C. P. 2012, RADMC-3D: A Multi-purpose Radiative Transfer Tool, Astrophysics Source Code Library, ascl:1202.015

Foreman-Mackey, D., Hogg, D. W., Lang, D., \& Goodman, J. 2013, PASP, 125,306

Isella, A., Chandler, C. J., Carpenter, J. M., Pérez, L. M., \& Ricci, L. 2014, ApJ, 788, 129

Kenyon, S. J., Dobrzycka, D., \& Hartmann, L. W. 1994, AJ, 108, 1872

Kraus, A. L., Andrews, S. M., Bowler, B. P., et al. 2015, ApJL, 798, L23

Kraus, A. L., \& Hillenbrand, L. A. 2009, ApJ, 704, 531

Kraus, A. L., Ireland, M. J., Cieza, L. A., et al. 2014, ApJ, 781, 20

Lynden-Bell, D., \& Pringle, J. E. 1974, MNRAS, 168, 603

MacGregor, M. A., Wilner, D. J., Czekala, I., et al. 2017, ApJ, 835, 17

Marley, M. S., Fortney, J. J., Hubickyj, O., Bodenheimer, P., \& Lissauer, J. J. 2007, ApJ, 655, 541

Ricci, L., Cazzoletti, P., Czekala, I., et al. 2017, AJ, 154, 24

Rosenfeld, K. A., Andrews, S. M., Hughes, A. M., et al. 2013, ApJ, 774, 16 Spiegel, D. S., \& Burrows, A. 2012, ApJ, 745, 174

White, R. J., \& Ghez, A. M. 2001, ApJ, 556, 265

Williams, J. P., \& Best, W. M. J. 2014, ApJ, 788, 59

Wolff, S. G., Ménard, F., Caceres, C., et al. 2017, AJ, 154, 26

Wu, Y.-L., Sheehan, P. D., Males, J. R., et al. 2017, ApJ, 836, 223

Yu, M., Evans, N. J., Dodson-Robinson, S. E., Willacy, K., \& Turner, N. J. 2017, ApJ, 841, 39

Zhou, Y., Herczeg, G. J., Kraus, A. L., Metchev, S., \& Cruz, K. L. 2014, ApJL, 783, L17 